\title{
Growth of bio sensor materials by physical vapor transport method
}

\author{
David Sachs, N. B. Singh, Ching-Hua Su*, Bradley Arnold, Brian M. Cullum, Fow-Sen Choa, \\ Tara Carpenter, and K. D. Mandal** \\ University of Maryland, Baltimore County, Baltimore, MD 21250 \\ * EM31, NASA Marshall Space Flight Center, Huntsville, AL 35812 \\ ***Indian Institute of Technology, Banaras Hindu University, Varanasi (UP) India
}

\begin{abstract}
Recently there is a big thrust on bio-inspired sensors and there has been a large rise in the investment and expectations for nanotechnology to meet these goals. For in situ sensor development materials deposition on substrate is essential part of device development. Physical vapor deposition (PVD), chemical vapor deposition (CVD) and molecular organic vapor deposition methods have developed for growth of semiconductor bulk and thin film growth with some modifications have been used for these materials. Oxides and other elements of VI group such as sulfides and selenides are key components in skins of many species. Growth of ordered structures containing these elements have been achieved by using PVD method. This paper describes effect of growth parameters during PVD growth on the quality of materials. Growth kinetics and mechanism will be discussed for the vertical and horizontal growth reactors. Since most of the efficient materials systems are multinary and in many cases non-congruent, PVD provides pathway to grow materials below melting temperature.
\end{abstract}

Keywords: Physical vapor transport, oxides, crystal, bioinspired, sensor, growth

\section{INTRODUCTION}

In the past century tremendous progress has been made in crystal growth science and technology. Bridgman, Czochralski, flux growth, Physical vapor transport (PVT), chemical vapor transport (CVT) and travelling solvent methods are commercial production of bulk single crystals. For nano, micro and thin film crystals molecular organic vapor epitaxial deposition, molecular beam epitaxial growth (MBE) and some techniques such as laser assisted techniques are frequently used. Out of all these techniques vapor transport method is widely used for both bulk and thin film crystals due to its flexibility specifically for incongruent materials. With increasing thrust on bio-inspired sensors, design of materials specially on surfaces which acts like heteroeptaxial growth has produced very complex morphologies. A large percentage of the living species such as animals, large and small fishes use piezo sensing. There is not much available in literature on the composition and morphology of the skin of animals especially fish which can provide information on the effect of fluid flow on their development. A group of researchers from Norway, Germany and the United states led by Giusi Minniti [1] studied and developed a correlation of impurities with the community present on the fish skin. They concluded after fish handling, the skin-mucus community exhibited structural similarity among the biological replicates. They suggested that there is a strong need of caution when interpreting community data due to the possible contamination of water-residing bacteria etc. This indicates importance of impurities during the growth of surface structures. Figure 1 shows, morphologies of salmon fish surfaces with very different morphologies obtained

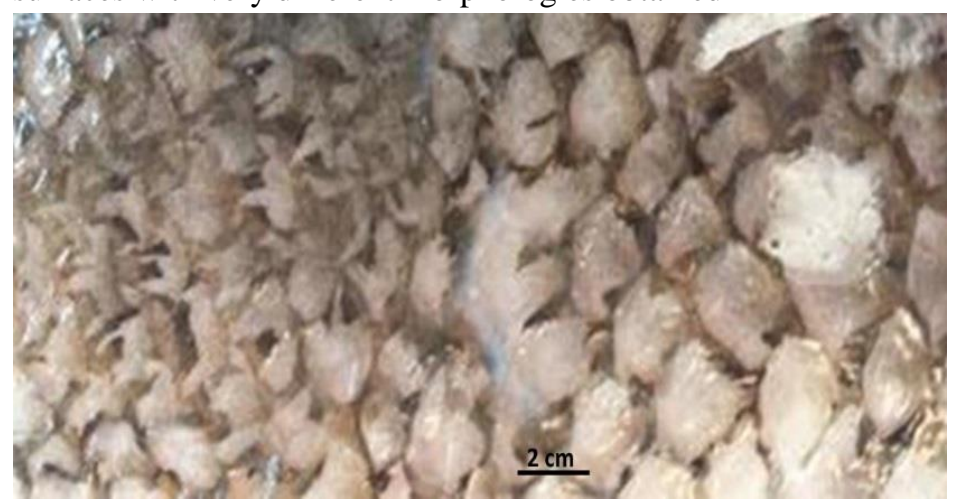

(a)

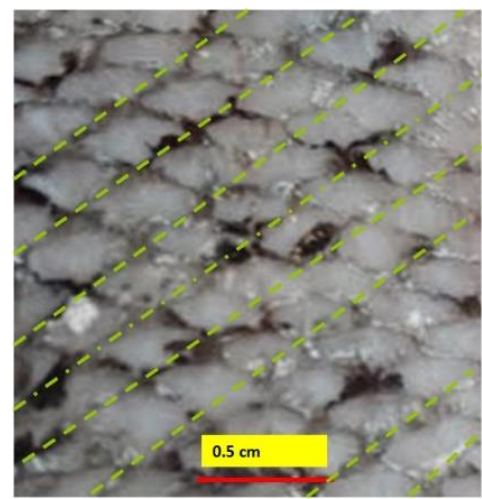

(b)

Figure 1. Irregular pattern on the skin of a typical pond salmon fish and (b) regular patterns on the skin of a typical salmon fish. Both were almost identical in size. 
from a pond and Atlantic Ocean. Figure 1 (b) indicates organized patterns (shown by lines) for a typical Atlantic salmon. The morphology of the skin is very similar to that of crystallographically arranged epitaxial growth on substrates. There is some hypothesis that turbulent flow affects the deposition in addition to slow etching of the surfaces removes the impurities from the surface. In addition to, heredity driven effects, fluid flow, effect of solute (salt in water), temperature $(\Delta \mathrm{T})$ in water and $\mathrm{pH}$ have pronounced effects on morphology of their skins. There have been tremendous efforts to develop meta materials and variety of structures to simulate skin materials (which act like sensors) of birds, fish and animals such as Scorpio for sensing UV to IR and whale for sensing RF wavelength regions. In this paper we will describe parameters of typical physical vapor transport growth and parameters affecting the growth rate and morphologies.

\section{EXPERIMENTAL METHODS AND RESULTS}

For the growth of biomaterials, containing large organic molecules such as proteins, crystallization method has been used extensively. However, growth of crystals larger than micrometer size has been challenge. In case of the complex biomaterials for various applications including for bone structures and bioinspired sensors such as shown in Fig. 2, it is extremely difficult to generate materials by crystallization due to multinary compositions and control of stoichiometry. Physical vapor transport (PVT) method has been successfully used in growth of industrially important class of materials for bulk crystals for hyperspectral imagers, lasers, sensors [2-6] and high-power RF devices and systems [7,9] in various geometry. A typical geometry for the vertical PVT is shown in Fig. 3. The source is in the bottom zone and cold zone is area where seed or substrate is placed for the growth. Among the parameters controlling growth by PVT method, such as temperature, vapor pressure, kinetics and properties of materials, fluid flow due to thermal profile (thermal convection) and solute driven (solutal convection) are most important parameters to grow and control the quality of materials.

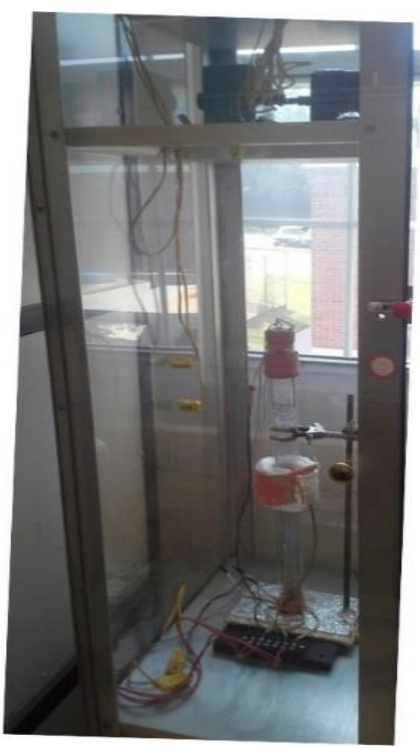

(a)

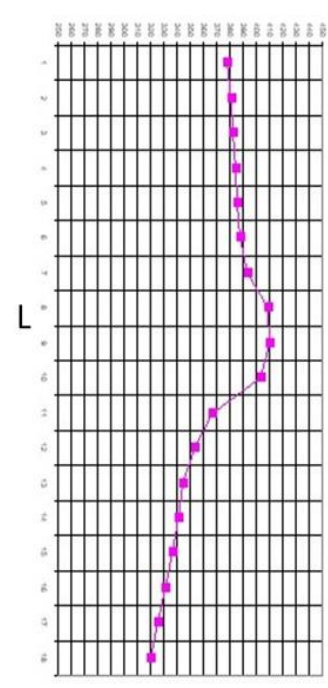

Temperature

(b)

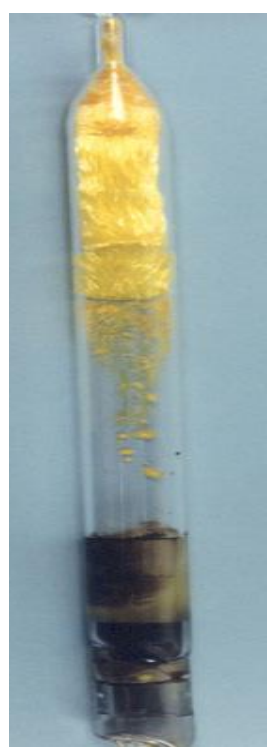

(c)

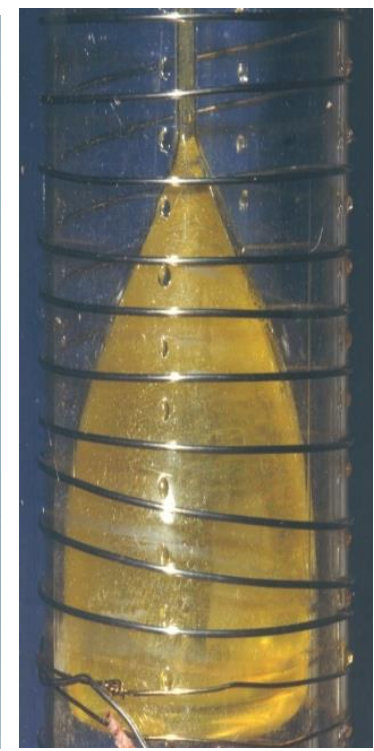

(d)

Figure 2. (a) Vertical two zone transparent furnace, (b) temperature scheme, (c) growth ampoule containing purified material by PVD and (d) seeded large bulk crystal growing in the upper zone of a two zone transparent furnace by PVD

For a very PVT growth geometry, such as shown in Fig. 3, growth models have been by substituting the general expression for shear stress into Newton's law of viscosity and integrating by applying the no-slip condition at the wall by deriving velocity profile across the circular duct geometry. The average velocity is defined as: $\mathrm{V}=\Delta \mathrm{P} \mathrm{a}^{2} /$ $8 \eta \mathrm{L}$, where $\Delta \mathrm{P}=\mathrm{P}_{\mathrm{S}}-\mathrm{P}_{\mathrm{c}}, \mathrm{P}_{\mathrm{S}}$ and $\mathrm{P}_{\mathrm{c}}$ are the vapor pressure at the source and crystal interfaces, $\eta$ is the viscosity, $8 \eta$ $\mathrm{L}$ a result which is Hagen-Poiseuille equation. 


$$
\alpha=\frac{\pi a^{4}}{8 \eta l}(P s-P e)
$$

Where $\alpha=$ Volume Flow Rate, $\eta=$ Viscosity. The number of moles $N$ per second through cross section is given by the equation:

$$
N=\frac{\pi a^{4}}{8 \eta l R}\left(\frac{P s^{2}-P c^{2}}{T s+T c}\right)
$$

Using this equation of number of moles growth velocity (without kinetic coefficient) can be derived as:

$$
V=\frac{9.6 a^{2} M}{\eta l \rho_{s}}\left(\frac{P s^{2}-P c^{2}}{T s+T c}\right)
$$

Where a and $l$ in $\mathrm{cm}, \mathrm{M}$ in $\mathrm{g} / \mathrm{mole}, \eta$ in Poise, $\rho_{\mathrm{s}}$ in $\mathrm{g} / \mathrm{cm}^{3}, \mathrm{Pc}$ and Ps in Torr, Tc and Ts in K.

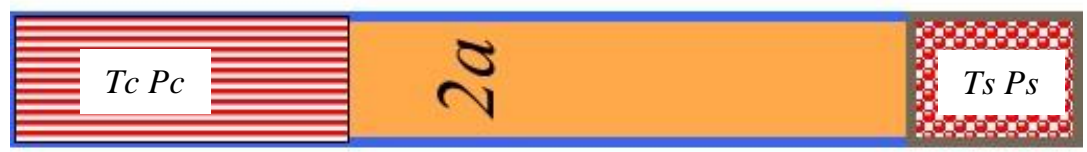

Figure 3. Schematics of horizontal PVD

Even in the simple geometry shown in Figure 2, formation of instabilities and cells due to thermal and solutal convection are almost impossible to avoid. Duval and coworkers [9] have performed extensive studies to develop science required documents (SRD) for PVT to justify role of microgravity to achieve pure diffusive growth. Although it is well established that $\mu \mathrm{g}$ gravity level environment is required for avoiding significant level flow, even $10^{-2}$ level gravity level affected (Fig. 4) the streamline flow in horizontal configuration. This indicates even $10^{-2}$ gravity level has significant effect in the diffusional growth.

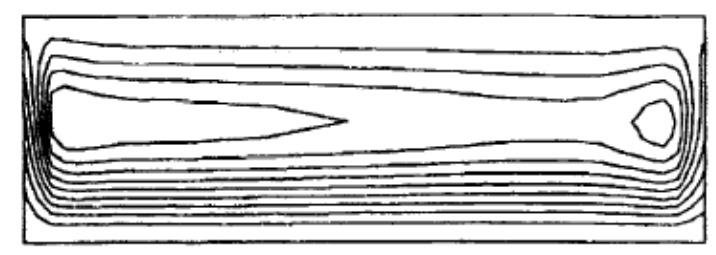

(a)

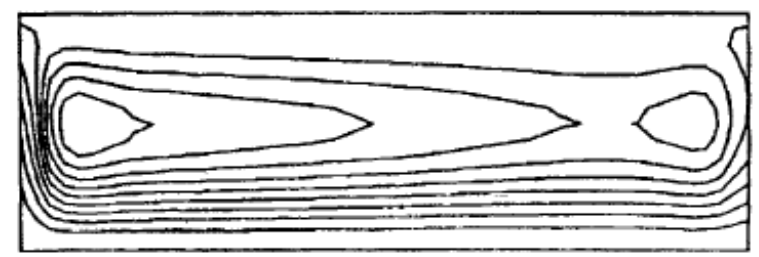

(b)

Figure 4. Streamline flow for gravity level of (a) $10^{-1} \mathrm{~g}$ and (b) $10^{-2} \mathrm{~g}$ in horizontal configuration [reference 9].

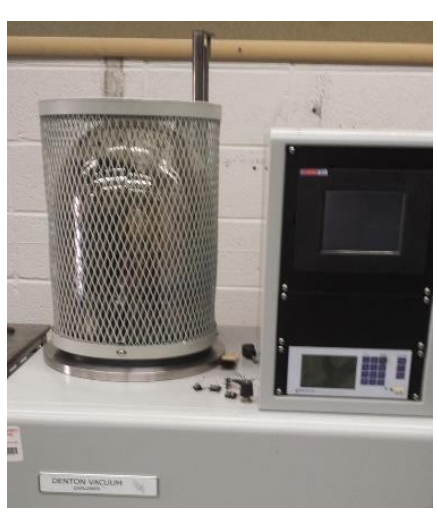

(a)

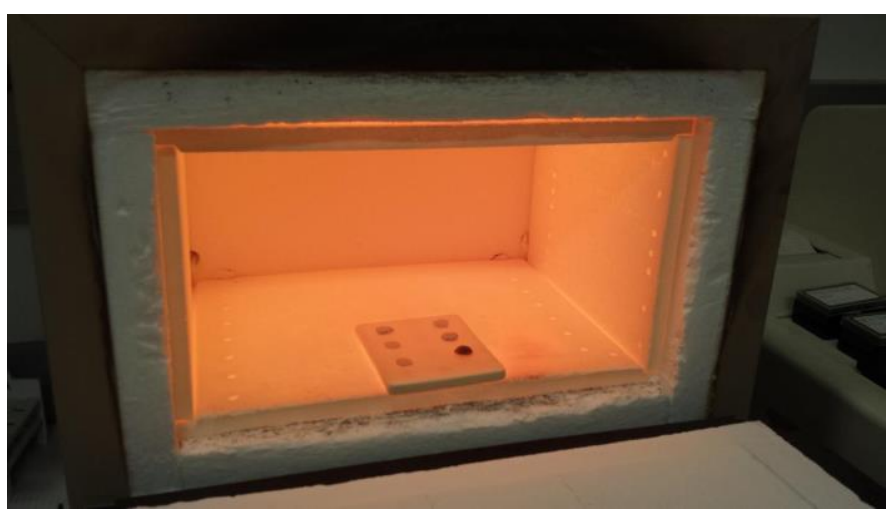

(b)

Figure 5. DENTON (a) growth PVT system and (b) growth chamber used in this PVT system 
The situation is very complex in large commercial reactors (Figure 5) where it is very difficult to control the diameter, path length, temperature of the source, sink, surface area of the seed or substrates. In addition, in large area growth chambers, fluid flow is complex. For systems such as chemical vapor deposition (CVD), MBE and MOCVD is even more complicated where forced flows are created during the evaporation of source materials. Fig. 6 shows the effect of fluid flow and pattern formation in (a) fire (Bottom hot top cold) and (b) a 15x 20 meter size (1.5 meter deep) pond created by steady dynamic flow air blowing from bottom. As shown in Fig. 6a an uncontrolled forest fire with solute (as results of fire) cause irregular double diffusive flows and cells of very large sizes. Careful observations at the suface of these cells show interface breaking due to inherited impurities and localized in the fire. To study the cell formations, we force air in a $15 \times 20$ meter pond approximately $30-40 \mathrm{~cm}$ below the surface with a $7.5 \mathrm{~mm}$ diameter pipe. As shown in Fig. 6b, we observed turbulent flow closer to the airflow tube and smaller patterns and cells far from the flow tube. In addition, white cells show area of heavy concentration area where solutal concentration driven cells dominate. These observations indicate that at both smaller and larger scale thermal and solutal convections play important role in fluid flow and hence in controlling the uniformity. In commercial DENTON reactors especially when sizes are very large, control of fluid flow as indicated in Fig $\mathbf{6}$ is very difficult. This explains why as the size of crystals increases, homogeneity of material decreases.

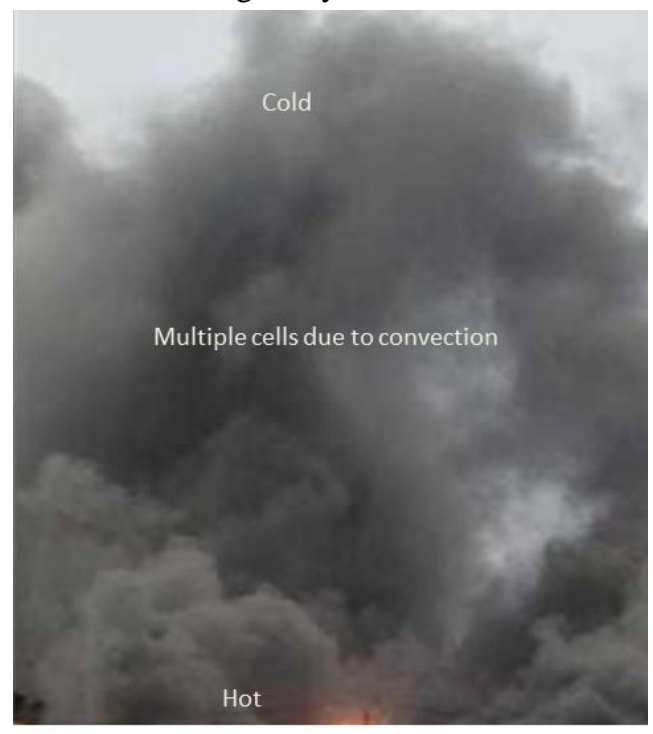

(a)

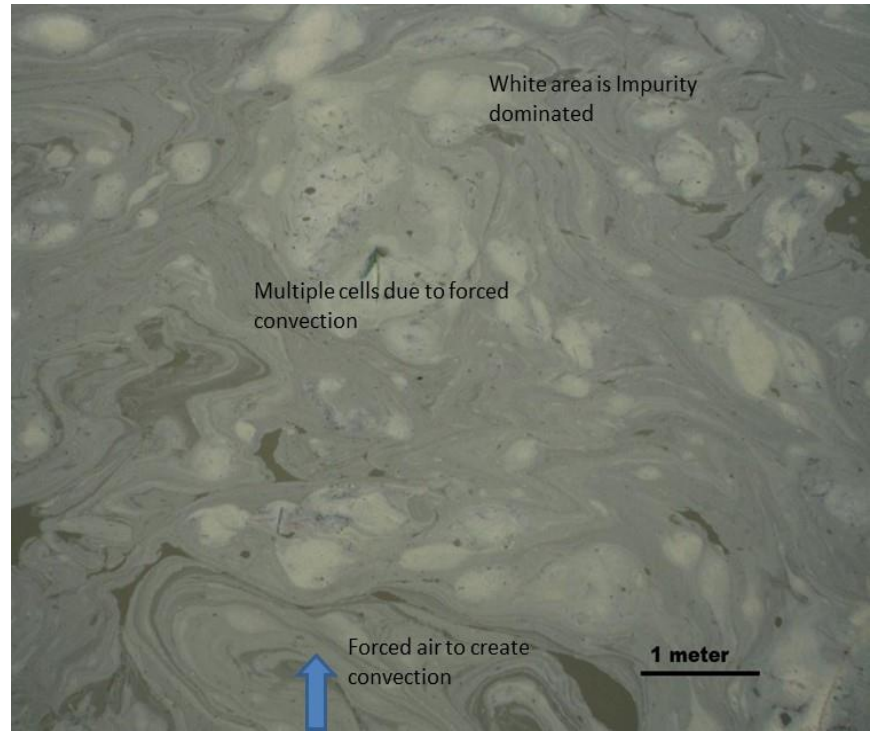

(b)

Figure 6. Effect of fluid flow and pattern formation in (a) fire (Bottom hot top cold) and (b) a 15x 20-meter size (1.5-meter-deep) pond created by steady dynamic flow air blowing from bottom

A detailed study has been performed [10] on the growth of pure and doped lead selenide for high temperature infrared detector. In this paper we show the results of two sets of experiments performed in the commercial DENTON to evaluate the effect of temperature driven fluid flow and its effect on morphology. Both experiments were performed with as supplied PbSe. No further purification was performed. In the first set of experiment, slow evaporation process was used by lowering the temperature of the hot zone by applying small power to the heater. In the second experiment, high temperature in hot zone was used to create turbulent flow. There was no attempt to control the substrate temperature. In both cases lightly etched (111) silicon substrates were used. All other parameters such as path length (distance between source and substrate), processing of substrate and grown material was identical. Fig.7 (b) and (c) shows the morphology of as grown materials. As shown in Fig.7 (b) and (c) there is a huge difference in morphology. material shown in Fig.7 (b) is uniform and show regular and uniform identical morphology while as morphology shown in Fig.7 (c) is very nonuniform and has PbSe precipitates embodied in the film. The difference in uniformity is due to flow difference during growth process. 

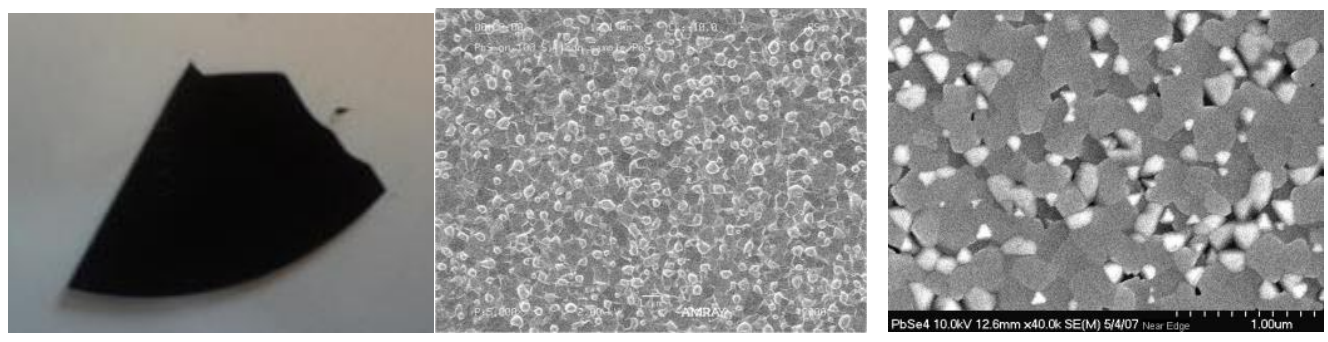

Figure 7. (a) film grown on Silicon substrate (b) morphology at low DT showing uniform morphology and (c) large DT showing different shaped grains and nonuniformity.

\section{SUMMARY}

A summary on the parameters of physical vapor transport growth has been presented. The main focus was on the effect of fluid flow on the morphology which controls the performance. Effect of thermal flow was evaluated qualitatively for the PVT growth DENTON evaporator for the growth of PbSe. We observed that convective flows can significantly change the growth morphology. For the growth of biosensor materials suitable for piezo and optical sensing especially oxides, sulfides and selenides, PVT an exciting technique. However, control of growth parameters is very important for achieving morphology.

\section{REFERENCES}

1. Giusi Minniti, Live Heldal Hagen, Davide Porcellato, Sven Martin Jørgensen, Phillip B. Pope, and Gustav Vaaje-Kolstad "The Skin-Mucus Microbial Community of Farmed Atlantic Salmon (Salmo salar)", Front Microbiol. 8: $2043\{2017\}$.

2. Ching Hua Su et al, "Contactless growth of ZnSe single crystals by Physical Vapor Transport", J. Crystal Growth, 213, 267-275 (2000).

3. Ching Hua Su et al, Vapor growth and characterization of ZnSeTe solid solutions”, J. Crystal Growth 216, 104$112(2000)$.

4. N. B Singh, R Mazelsky and M. E Glicksman "Evaluation of transport conditions during PVT growth of optoelectronic crystals", AIAA J. Thermo Phys. 4, 126, (1989).

5. N.B. Singh, M. Gottlieb, T. Henningsen, G.J. Davies. Effect of temperature gradient on the quality of mercurous chloride crystals, J. Crystal Growth, 96, 969 (1989).

6. N. B. Singh, Ching Hua Su, Bradley Arnold and Fow-Sen Choa,"Optical and morphological characteristics of ZnS-ZnSe solid solution" Optical Materials 60, 474-480 (2016)

7. Narsingh B. Singh, B. Wagner, Andre Berghmans, David J. Knuteson, Sean McLaughlin, David Kahler, Darren Thomson and Matthew King, "( $\mathrm{SiC})_{\mathrm{x}}(\mathrm{AlN})_{1-\mathrm{x}}$ solid-solution substrates for high temperature and high-power devices", J. Crystal Growth and Design, 10(8), 3508-3515 (2010).

8. Narsingh Bahadur Singh, Christopher Cooper, Pietro Strobbia, Narasimha Prasad, Ching Hua Su, Bradley Arnold, Fow-Sen Choa, "Nanomorphology and performance of pure and doped lead selenide for infrared detector," Opt. Eng. 56(7), 077106 (2017)

9. Christopher Mennetrier, W. M. B. Duval and N. B. Singh, "Physical vapor transport of mercurous chloride under nonlinear thermal profile" NASA Technical Memorandom 105920, November 1992.

10. N. B. Singh, Ching Hua Su, Christopher Cooper, Sonali Saraf, Stacey Sova, Bradley Arnold, Fow-Sen Choa, "Nanomorphology and performance of pure and doped lead selenide" (To be communicated) 